\title{
Inhibición del crecimiento in vitro de Fusarium oxysporum Schltdl., con extracto de Argemone ochroleuca Sweet (Papaveraceae)
}

\author{
In vitro growth inhibition of Fusarium oxysporum Schltdl., \\ with Argemone ochroleuca Sweet (Papaveraceae) extract
}

\author{
Rosa Adriana Juárez-García , Diana Sanzón-Gómez ${ }^{1}$, Luis Felipe Ramírez-Santoyo ${ }^{1}$ \\ Jorge Eric Ruíz-Nieto ${ }^{1}$, Jesús Hernández-Ruiz ${ }^{1^{*}}$
}

${ }^{1}$ División de Ciencias de la Vida, Universidad de Guanajuato, Km 9 carretera Irapuato-Silao, Ex Hacienda El Copal, 36500, Irapuato, Guanajuato, México.

*Autor para correspondencia: hernandez.jesus@ugto.mx

Fecha de recepción:

28 de enero de 2020

Fecha de aceptación:

6 junio de 2020

Disponible en línea:

5 de agosto de 2020

Este es un artículo en acceso abierto que se distribuye de acuerdo a los términos de la licencia Creative Commons.

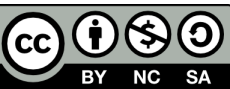

Reconocimiento-

NoComercial-CompartirIgual

4.0 Internacional
RESUMEN

El hongo Fusarium oxysporum Schltdl. (Ascomycetes) es causante de enfermedades en la fresa. Los fungicidas sintéticos son las principales herramientas para su control; sin embargo, éstos causan severos daños al medio ambiente. Una alternativa es el uso de compuestos de origen botánico con actividad antifúngica. El objetivo del presente estudio fue evaluar el efecto de extracto de Argemone ochroleuca Sweet (Papaveraceae) contra F. oxysporum en condiciones in vitro. Se recolectaron plantas de fresa con síntomas característicos de secadera; los hongos aislados se purificaron y caracterizaron morfológica y molecularmente. La inhibición del crecimiento se evaluó utilizando tres concentraciones del extracto de A. ochroleuca (13, 23 y 31\%). Los aislados obtenidos se identificaron como F. oxysporum. La inhibición del crecimiento fue de $66 \%$ al noveno día, con extracto a 13\%, mientras que las concentraciones de 23 y $31 \%$ inhibieron en su totalidad el crecimiento a partir del tercer día de evaluación.

\section{PALABRAS CLAVE}

Secadera de la fresa, extracto vegetal, chicalote.

\section{ABSTRACT}

The fungus Fusarium oxysporum Schltdl. (Ascomycetes) causes strawberry diseases. Synthetic fungicides are primarily used for their control; however, these fungicides have a negative impacts on the environment. An alternative control strategy is the use of antifungal compounds of botanical origin. The objective of this study was to evaluate extracts of Argemone ochroleuca Sweet (Papaveraceae) on F. oxysporum viability under in vitro conditions. Strawberry plants with characteristic foliar disease symptoms were collected, the isolated fungi were purified and characterized both morphologically and molecularly. Growth inhibition was evaluated using three concentrations of the extract $(13,23$, and $31 \%)$. The isolates were identified as $F$. oxysporum. Their growth inhibition was by $66 \%$ on the ninth day using $13 \%$ of the extract, whereas the 23 and $31 \%$ concentrations inhibited the growth of F. oxysporum entirely on the third day of evaluation.

KEYWORDS

Strawberry disease, vegetal extract, chicalote. 


\section{INTRODUCCIÓN}

La fresa (Fragaria ananassa (Duchesne ex Weston) Duchesne ex Rozier, Rosaceae) es el cultivo de frutillas más importante en México, en términos económicos. Este país es el cuarto productor a nivel mundial, con 458,972 toneladas anuales, lo que representa $4.08 \%$ del total global (FAOSTAT 2018). A nivel nacional, Michoacán, Baja California y Guanajuato son las entidades de mayor producción, al aportar más de $96 \%$ (379,552 ton/año) (SIAP 2018). Cabe señalar que el cultivo de fresa presenta diversas enfermedades causadas por microorganismos, principalmente por infección con hongos, como la mancha foliar (Alternaria alternata (Fries) Keissler), la antracnosis (Colletotrichum fragariae Brooks y C. acutatum Simmonds) y la pudrición de las raíces negras (Fusarium sp., Verticillium sp. y Rhizoctonia sp.) (Chidichima et al. 2018; Bárcenas-Santana et al. 2019).

En este sentido, el ataque de ciertos hongos, como Fusarium sp., Rhizoctonia sp., Verticillium sp., y Phytophthora sp., agentes causantes de la enfermedad conocida como "secadera", así como otras enfermedades coronarias y de raíz asociadas a estos microorganismos, se consideran un grave problema para la producción agrícola de la fresa, ya que les generan pérdidas económicas a los productores (De los Santos et al. 2003).

Entre el complejo de hongos causantes de la "secadera", Fusarium oxysporum f. sp. fragariae (Fof) es el de mayor importancia, por su amplia distribución, así como por la susceptibilidad de las variedades de fresa, la magnitud de los daños ocasionados y la presencia activa durante todo el ciclo de cultivo, tanto en vivero como en plantaciones comerciales, lo que provoca una disminución considerable en el rendimiento y genera enormes pérdidas económicas a los productores (Ceja-Torres et al. 2008).

La presencia de F. oxysporum y la alta contaminación por insumos agrícolas en los suelos ocasionan que la productividad del cultivo sea altamente inestable entre años y localidades (León-López et al. 2014). Desde la década de 1990, este hongo es el más frecuente, pues se presenta desde etapas tempranas del cultivo (trasplante) y causa pérdidas mayores a $50 \%$, equivalente a 7.5 ton ha $^{-1}$ (Castro y Dávalos 1990); esta situación persiste, ya que Mariscal-Amaro et al. (2017) reportan que la frecuencia de esta especie en el cultivo de fresa en Guanajuato fue de 54\% en el ciclo PV/2014 y de 60\% en el ciclo PV/2015.

Para controlar este patógeno, se utilizan fungicidas sintéticos, como el bromuro de metilo, de amplio espectro y el cual se aplicaba en el suelo como parte de la preparación antes de la siembra de la fresa, cuyo uso, sin embargo, se ha prohibido, debido a las restricciones establecidas por las regulaciones internacionales, por considerarse que daña la capa de ozono (Ajwa et al. 2003). Otros productos químicos utilizados son la cloropicrina (Pic) y el 1,3-dicloropropeno (1,3-D) (López-Medina et al. 2007; Zhang et al. 2019), los cuales, de acuerdo con el Registro Sanitario de Plaguicidas, Nutrientes Vegetales y LMR de México, cuentan con la categoría toxicológica I y IV, respectivamente (COFEPRIS 2020).

Sin embargo, el control basado en fungicidas químicos implica el desarrollo de resistencia de los patógenos, así como problemas ambientales y el impacto de los residuos sobre organismos benéficos, lo que representa un riesgo tanto para la sanidad del suelo, como para la salud de los consumidores de fresa (Pedraza et al. 2010; Tortora et al. 2012).

Esto evidencia la necesidad de nuevos productos, basados en metabolitos secundarios de plantas, que sean utilizados en distintas estrategias de control (Mdee et al. 2009); dentro de éstos se encuentran los extractos botánicos, que pueden emplear especies del género Argemone (Sharma et al. 2017) para el control de plagas y enfermedades en el sector agrícola (Hernández-Ruiz et al. 2020; Gerwick y Sparks 2014; Gahukar 2016). Los metabolitos poseen diversas características que los convierten en una opción viable de control, dado que poseen actividad biológica en contra de patógenos agrícolas como hongos, bacterias y virus; son biodegradables, por lo que no afectan al equilibrio ambiental; no tienen restricciones toxicológicas y reducen costos de producción (Isman y Seffrin 2014). Al menos 45 alcaloides bencilisoquinolínicos son sintetizados en los diversos órganos de la planta y tienen capacidad de inhibir el crecimiento de bacterias, hongos y virus, principalmente protopinas, benzofenantridinas $\mathrm{y}$ protoberberinas (Brahmachari et al. 2013; Roberts y Wink 1998). 
Plantas del género Argemone se han evaluado para el control de plagas y enfermedades, esto incluye su incorporación al suelo, donde inhibieron la esporulación y crecimiento de cinco hongos Macrophomina phaseolina (Tassi) Goid, Fusarium oxysporum Schltdl., Rhizoctonia solani Kuhn, Phyllosticta phaseolina Sacc., y Sclerotium rolfsii Sacc. (Rizvi et al. 2012). Extractos acuosos de raíz y partes vegetativas mostraron efectos tóxicos para el control de nematodos como Meloidogyne incognita (Kofoid \& White) y M. javanica (Treub) Chitwood en cultivos de soya (Glycine max (L.) Merr.) y berenjena (Solanum melongena L.) (Gahukar 2016). En gusano cogollero (Spodoptera frugiperda (J. E. Smith)), extractos de Argemone ochroleuca Sweet causan reducción en la alimentación y crecimiento de las larvas, así como aumento de la mortalidad en 10\% (Martínez et al. 2017). Se reporta que alcaloides terciarios y cuaternarios aislados de $A$. ochroleuca inhiben completamente la germinación de esporas de Fusarium udum E.J. Butler y Helminthosporium sp., a una concentración de 200 ppm, y de Alternata alternata (Fr.) Keissl. y Colletotrichum musae (Berk. \& M.A. Curtis) Arx a 600 y 800 ppm, respectivamente (Singh et al. 2010). Por lo anterior, el objetivo de la presente investigación fue llevar a cabo pruebas preliminares de inhibición del crecimiento in vitro de F. oxysporum con extracto de A. ochroleuca.

\section{Materiales y métodos}

\section{Caracterización morfológica y molecular del hongo}

Durante el ciclo agrícola primavera-verano de 2017, se recolectaron 10 plantas de fresa del cultivar Camino Real, con síntomas característicos de secadera de la fresa (marchitez, enanismo y clorosis), en parcelas de tres localidades de Irapuato, Guanajuato, México. Los hongos aislados se purificaron mediante la técnica de cultivos monospóricos y por punta de hifa en medio Papa-Dextrosa-Agar (PDA, BD Bioxon®), $39 \mathrm{~g} \mathrm{~L}^{-1}$ en agua destilada con la adición de $0.25 \%$ de ácido láctico. De cada planta del tejido de raíz se obtuvieron cuatro aislados puros.

Para la clasificación del género Fusarium se observó el crecimiento micelial y la pigmentación del medio de cultivo, $\mathrm{y}$, mediante preparaciones temporales, la caracterización de microconidos, macroconidios y clamidosporas. Las características culturales y morfológicas se compararon con las descripciones de Nelson et al. (1983). Se seleccionó un aislado de cada localidad y se sembró en medio PapaDextrosa-Agar (PDA, BD Bioxon $\left.{ }^{\circledR}\right), 20 \mathrm{~g} \mathrm{~L}^{-1}$ en agua destilada. Los aislados seleccionados se emplearon para la identificación molecular mediante la amplificación de la región espaciadora transcrita (ITS). La extracción de ADN de cada aislado se efectuó mediante el protocolo de Dellaporta et al. (1983), con la adaptación del buffer de extracción (100 mM Tris-HCI pH:8.0; 50 mM EDTA pH:8.0; 500 mM N aCI; $1.25 \%$ SDS; $0.38 \mathrm{~g}$ NaHSO3 por cada $100 \mathrm{ml}$ de buffer). Para la PCR se usó el oligonucleótido sentido ITS1-F (5'-CTTGGTCATTTAGAGGAAGTAA-3') (Gardes y Bruns 1993) y el oligonucleótido antisentido ITS4 (5'-TCCTCCGCTTATTGATATGC-3'). Se usó la mezcla de reacción descrita por White et al. (1990). La amplificación se hizo con un termociclador SimpliAmp Thermal Cycler A24811 (Thermo Fisher Scientific ${ }^{\mathrm{TM}}$, EUA), con el programa de amplificación empleado por Ceja-Torres et al. (2008): 1 ciclo $95^{\circ} \mathrm{C}, 1 \mathrm{~min}$; 30 ciclos a $95^{\circ} \mathrm{C}, 1 \mathrm{~min} ; 57^{\circ} \mathrm{C}, 1 \mathrm{~min} ; 72^{\circ} \mathrm{C}, 1 \mathrm{~min} ; 1$ ciclo a $72^{\circ} \mathrm{C}, 12 \mathrm{~min}$. Los productos se purificaron con el Kit FastDNA SPIN (FastDNA TM, EUA), de acuerdo con el protocolo del fabricante. Los productos purificados se secuenciaron en un ABI Prism 3130 Genetic Analyzer (Applied Biosystems ${ }^{\mathrm{TM}}$, EUA). Las secuencias obtenidas se compararon y alinearon con la base de datos del Banco de Genes del National Center for Biotechnology Information (NCBI), con BLAST y Clustal W de DNASTAR Ver. 3.1 (DNASTAR, Inc.).

\section{Obtención del extracto de Argemone ochroleuca (chicalote)}

En Copal, Irapuato, Guanajuato, México, se ubicaron plantas herbáceas de ciclo anual; de vida corta; glaucas; con látex amarillo; vástagos provistos de espinas rectas, blanquecinas, ampliamente espaciadas, de longitudes diversas, perpendiculares a la superficie en la que se originan; con tallo simple o ramificado en la parte superior; hojas con frecuencia dispuestas en roseta en la parte basal de la planta, de oblanceoladas a elípticas; botones florales cilindráceos, con al menos tres espinas finas sobre cada sépalo; cuernos apicales divergentes, rollizos o algo aplanados y triangulares-subulados; flores de pétalos de color crema, 
obovados u obcuneiformes a elípticos, con filamentos y anteras amarillas, estigma de color púrpura (Figura 1). Estas plantas se colectaron e identificaron como Argemone ochroleuca con base en la descripción botánica de Calderón (1991) y Rzedowski y Rzedowski (2001).

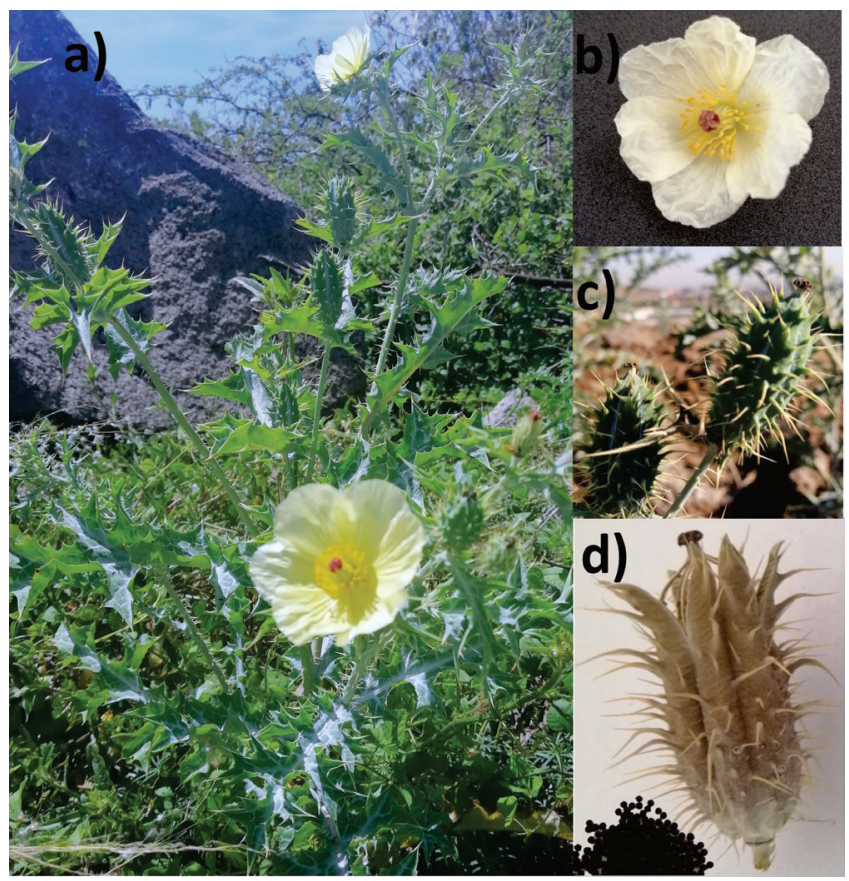

Figura 1. Argemone ochroleuca colectada en Irapuato, Guanajuato, México; a) Planta con flor y tallos ramificados; b) flor; c) botones florales; d) frutos y semillas.

Las plantas fueron cortadas con tijeras de podar hasta dejar ramas de $2 \mathrm{~cm}$ sobre el eje principal. Posteriormente, se pesaron $250 \mathrm{~g}$ de material vegetal fresco, en una balanza analítica (Marca XB modelo 220A). Al material se le practicó un prelavado con solución dehipoclorito de sodio a $0.5 \%$ por $2 \mathrm{~min}$, y luego cuatro lavados con agua destilada estéril. Después, se agregaron $250 \mathrm{~mL}$ de etanol a $96 \%$ y se molieron hasta obtener una mezcla homogénea. La mezcla se filtró utilizando cuatro capas de gasa estéril y luego se utilizó papel filtro estéril para la última filtración. El extracto total obtenido se dispuso en frascos de vidrio color ámbar para llevarse a refrigeración a $4^{\circ} \mathrm{C}$ hasta su utilización (Ochoa et al. 2012).

\section{Evaluación de la inhibición del crecimiento radial}

La evaluación de la actividad del extracto contra el hongo se hizo por medio de la medición del halo del crecimiento del hongo respecto al control negativo. Se preparó Papa-Dextrosa-Agar (PDA, BD Bioxon $\left.{ }^{\circledR}\right)$, como indica el fabricante: $39 \mathrm{~g} \mathrm{~L}^{-1}$ agua destilada, se esterilizó a $121^{\circ} \mathrm{C}$ por $15 \mathrm{~min}$; se ajustó el pH a 5.0 con ácido tartárico antes de vaciar $20 \mathrm{~mL}$ de PDA en las cajas de Petri que contenían las concentraciones de extracto de A. ochroleuca de los tres tratamientos (13, 23 y $31 \%)$. Se dejaron solidificar para posteriormente ser inoculadas con círculos de $3 \mathrm{~mm}$ de diámetro del hongo fitopatógeno, de ocho días de incubación. Los círculos se colocaron en el centro de la caja, se incubaron a $26 \pm 1^{\circ} \mathrm{C}$. Para la evaluación de la inhibición del crecimiento de los hongos se midió el diámetro del crecimiento micelial con un vernier digital, a los 3, 6, 9 y 12 días después de la inoculación. Para todas las concentraciones, al igual que para el control positivo, se hicieron cuatro repeticiones.

El porcentaje de inhibición del crecimiento se calculó como se indica a continuación:

$\%$ de inhibición $=\frac{\begin{array}{c}\text { diámetro micelial del testigo }- \text { diámetro } \\ \text { micelial del tratamiento }\end{array}}{\text { diámetro micelial del testigo }} \times 100$

Los datos de crecimiento de los halos fueron analizados con un diseño experimental completamente al azar con 4 repeticiones. Cada repetición constó de tres cajas por evaluación y una prueba de comparación de medias por DMS ( $\mathrm{p} \leq 0.01)$, con el paquete estadístico SAS 9.0. (SAS 2002).

\section{Resultados Y Discusión}

De las colectas efectuadas se caracterizaron tres cepas de F. oxysporum, las cuales produjeron colonias rosas, que se tornaron violetas con la edad; microconidios ovoides y hialinos producidos en fiálides cortas; macroconidios ligeramente curvados con 3 a 5 septos, y clamidosporas terminales o intercalares (Figura 2). Estas características concuerdan con lo reportado por Mariscal-Amaro et al. (2017), quienes describen aislados de F. oxysporum, de cultivos de fresa procedentes de Guanajuato, con características culturales tales como pigmentaciones de color rosa, salmón, rojo, magenta y violeta, las cuales se tornaron más oscuras con la edad, y, en algunos casos, con abundante micelio algodonoso y esparcido en la caja Petri, y con la descripción morfológica de Ceja-Torres et al. (2008), 
quienes describen aislados provenientes de fresa de Michoacán, como una especie fungosa que conforma colonias rosa púrpura, que se tornaron violeta, con microconidios ovoides producidos en fiálides cortas, macroconidios ligeramente curvados con septos.

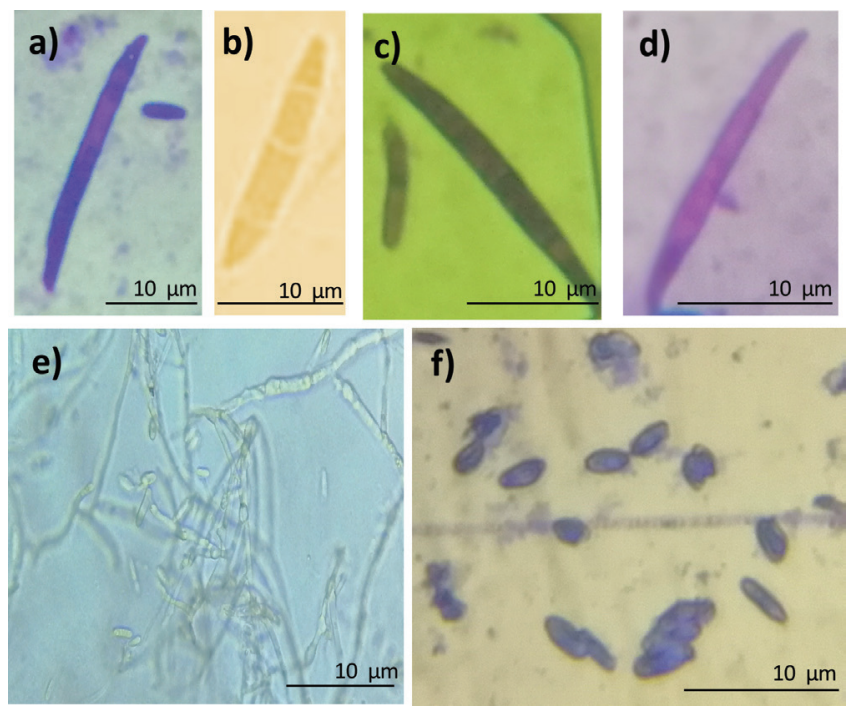

Figura 2. Estructuras morfológicas de Fusarium oxysporum en aumento inmersión (100x); a-d) micronidios, e) micelio, f) clamidiosporas.

Las secuencias de dos aislados tuvieron una homología de 99\% con las accesiones DQ979012 (Versión DQ979012.2); DQ452072 (Versión DQ452072.1), las cuales Ceja-Torres et al. (2008) reportan con una homología de $99 \%$ y valor de similitud de 959 con la mayoría de las secuencias de Fusarium oxysporum, raza F-H 6.5, disponibles en el Genbank.

El crecimiento radial del hongo se observó en el testigo y en el tratamiento con la concentración de extracto de Argemone a 13\%. Los tratamientos con la concentración de 23 y 31\% no mostraron desarrollo del hongo, lo que evidenció el efecto fungicida del extracto sobre el patógeno evaluado. Este efecto se atribuye al compuesto dehidrocoridalmina (Singh et al. 2009), uno de los 45 alcaloides reportados en los diversos órganos de las plantas del género Argemone (Brahmachari et al. 2013). Sin embargo, para A. ochroleuca se reporta la presencia de 14 alcaloides del tipo isoquinolinas, dentro de los cuales no se encuentra dehidrocoridalmina; por su parte, la dihidrochelerithrina (C21H19NO4) y dihidrosanguiranina (C20H15NO4) son los más abundantes en las semillas y en el tejido vegetativo de la especie, cuya actividad biológica está relacionada con actividad antibacteriana y molusquicida, los que la convierte en una fuente potencial de alcaloides para usos agrícolas (Hernández-Ruiz et al. 2020).

Los extractos de A. ochroleuca a 23 y $31 \%$ presentaron una inhibición de $100 \%$ al tercer día, en tanto que la concentración de $13 \%$ presentó $51 \%$ de inhibición al tercer día, y se incrementó hasta $66 \%$ al día nueve (Cuadro 1). Se ha reportado una disminución en la capacidad de infección de los hongos de los géneros Fusarium, Phyllocsticta y Sclerotium, al incorporar plantas de Argemone sp. al suelo (Siddiqui et al. 2002; Rizvi et al. 2012; Gahukar 2016). Por otra parte, se han efectuado pruebas in vitro para el control de F. oxysporum con extractos de Nicotiana glauca Graham y Shinus molle L., donde no se registró efecto de inhibición (Ochoa et al. 2012).

Cuadro 1. Inhibición del crecimiento micelial de $F$. oxysporum por distintas concentraciones de Argemone ochroleuca

\begin{tabular}{cllll}
\hline \multirow{2}{*}{$\begin{array}{c}\text { Concentración } \\
\text { del extracto (\%) }\end{array}$} & \multicolumn{4}{c}{ Porcentaje de inhibición en } \\
& día 3 & día 6 & día 9 & día 12 \\
\hline 0 & 0 & 0 & 0 & 0 \\
13 & 51.50 & 63.86 & 66.54 & 64.48 \\
23 & 100 & 100 & 100 & 100 \\
31 & 100 & 100 & 100 & 100 \\
\hline
\end{tabular}

La concentración de extracto tuvo un efecto estadísticamente significativo en el crecimiento del hongo, al formarse tres grupos diferentes. Los mayores efectos se presentaron al utilizar las concentraciones de $23 \mathrm{y}$ $31 \%$, donde no hubo un halo de crecimiento (Cuadro 2). Esto demuestra que las plantas que contienen compuestos con propiedades antifúngicas afectan a patógenos de una forma directamente proporcional: el aumento de la concentración del extracto evaluado incrementa la inhibición del hongo (Villa-Martínez et al. 2015). Este efecto también fue reportado por De Rodríguez et al. (2007), quienes evaluaron extractos etanólicos de Flourensia microphylla (A.Gray) S.F.Blake, F. cernua DC y F. retinophylla S.F.Blake contra F. oxysporum, donde, según el aumento de concentración del extracto de la planta 10, 100, 500, 1000 y $1500 \mu \mathrm{l}^{-1}$, aumentó la inhibición en el crecimiento micelial en 20, $75,95,100$ y $100 \%$, respectivamente. 
Cuadro 2. Comparación múltiple de medias para los halos de crecimiento (centímetros) de F. oxysporum, de acuerdo con las diferentes concentraciones de extracto de A. ochroleuca.

\begin{tabular}{cllll}
\hline \multirow{2}{*}{ Concentración del extracto (\%) } & \multicolumn{4}{c}{ Porcentaje de inhibición en F. oxysporum } \\
& día 3 & día 6 & día 9 & día 12 \\
\hline 0 & $2.52 \mathrm{a}^{*} \pm 0.02$ & $4.15 \mathrm{a} \pm 0.10$ & $6.42 \mathrm{a} \pm 0.8$ & $7.25 \mathrm{a} \pm 0.25$ \\
13 & $1.22 \mathrm{~b} \pm 0.75$ & $1.50 \mathrm{~b} \pm 0.15$ & $2.15 \mathrm{~b} \pm 0.15$ & $2.5 \mathrm{~b} \pm 0.22$ \\
23 & $0.0 \mathrm{c} \pm 0.0$ & $0.0 \mathrm{c} \pm 0.0$ & $0.0 \mathrm{c} \pm 0.0$ & $0.0 \mathrm{c} \pm 0.0$ \\
31 & $0.0 \mathrm{c} \pm 0.0$ & $0.0 \mathrm{c} \pm 0.0$ & $0.0 \mathrm{c} \pm 0.0$ & $0.0 \mathrm{c} \pm 0.0$ \\
\hline
\end{tabular}

Nivel de significancia $=0.05$. ${ }^{*}$ Letras diferentes en una misma columna indican diferencia significativa. Cada valor corresponde al promedio de cuatro repeticiones más desviación estándar.

\section{CONCLUSIONES}

Extracto de Argemone ochroleuca presenta propiedades antifúngicas. Concentraciones de 23 y $31 \%$ inhibieron en su totalidad el crecimiento de Fusarium oxysporum aislado del cultivo de fresa en Irapuato, Guanajuato, México.

\section{AGRADECIMIENTOS}

Esta investigación fue apoyada por la Secretaría de Innovación, Ciencia y Educación Superior (SICES), del estado de Guanajuato, como parte del proyecto "Determinación geoespacial de áreas susceptibles a plagas y enfermedades del cultivo de fresa en Guanajuato" (oficio DRF/860/2019). 


\section{LITERATURA CITADA}

Ajwa HA, Klose S, Nelson SD, Minuto A, Gullino ML, Lamberti F, Lopez-Aranda JM. 2003. Alternatives to methyl bromide in strawberry production in the United States of America and the Mediterranean region. Phytopathologia Mediterranea 42(3): 220-244.

Bárcenas-Santana D, Guillén-Sánchez D, YazmínBasaldua C, Ramos-García ML, Valle-de la Paz M. 2019. Etiología de la secadera de la fresa (Fragaria spp.) en Morelos, México. Revista Mexicana de Fitopatología 37(3): 1-10. https://doi.org/10.18781/R.MEX.FIT.1904-5

Brahmachari G, Gorai D, Roy R. 2013. Argemone mexicana: Chemical and pharmacological aspects. Revista Brasileira de Farmacognosia 23(3): 559-575. https://doi. org/10.1590/S0102-695X2013005000021

Calderón RG. 1991. Familia Papaveraceae. In: Rzedowski J, editor. Flora del Bajío y de regiones adyacentes. Fascículo 54. Pátzcuaro, Instituto de Ecología, A.C., Centro Regional del Bajío. P. 185-188.

Castro FJ, Dávalos G. 1990. Etiología de "la secadera" o pudrición de la raíz y corona de la fresa en Irapuato, Guanajuato. Revista Mexicana de Fitopatología 8: 8086. https://doi.org/10.18781/r.mex.fit.1806-1

Ceja-Torres LF, Mora-Aguilera G, Téliz D, Mora-Aguilera A, Sánchez-García P, Muñoz-Ruíz C, La TorreAlmaraz C. 2008. Ocurrencia de hongos y etiología de la secadera de la fresa con diferentes sistemas de manejo agronómico. Agrociencia 42(4): 451-461.

Chidichima LDS, Nozaki MDH, Hendges C, Gaias WL. 2018. Growth and etiology strawberry anthracnosis causal. Acta Iguazu 7(4): 24-34.

[COFEPRIS] Comisión Federal para la Protección contra Riesgos Sanitarios. [internet]. 2020. Consulta de Registros Sanitarios de Plaguicidas, Nutrientes Vegetales y LMR. [cited 2020 May 01]. Disponible en: http://siipris03.cofepris.gob.mx/Resoluciones/ Consultas/ConWebRegPlaguicida.asp.

Dellaporta SL, Wood J, Hicks JB. 1983. A plant DNA minipreparation: Version II. Plant Molecular Biology Reporter 1(4): 19-21. https://doi.org/10.1007/ BF02712670

De los Santos B, Barrau C, Romero F. 2003. Strawberry fungal diseases. Journal of Food Agriculture and Environment 1(3): 129-132.

De Rodríguez DJ, Hernández-Castillo D, Angulo-Sánchez JL, Rodríguez-García R, Villarreal Quintanilla JA,
Lira-Saldivar RH. 2007. Antifungal activity in vitro of Flourensia spp. extracts on Alternaria sp., Rhizoctonia solani, and Fusarium oxysporum. Industrial Crops and Products 25(2): 111-116. https://doi.org/10.1016/j. indcrop.2006.08.007

[FAOSTAT] Statistical databases. Food and Agriculture Organization of the United Nations. [internet]. 2018. [cited 2019 Oct 19]. Disponible en: http://www.fao. org/faostat/es/?\#data/QC.

Gahukar RT. 2016. Plant-derived products in crop protection: Effects of various application methods on pests and diseases. Phytoparasitica 44(3): 379-391. https:// doi.org/10.1007/s12600-016-0524-3

Gardes M, Bruns TD. 1993. ITS primers with enhanced specificity for basidiomicetes application to the identification of mycorrhizae and rusts. Molecular Ecology 2(2): 113-118. https://doi.org/10.1111/j.1365-294x.1993. tb00005.x

Gerwick BC, Sparks TC. 2014. Natural products for pest control: An analysis of their role, value and future. Pest Management Science 70(8): 1169-1185. https://doi. org/10.1002/ps.3744

Hernández-Ruíz J, Bernal J, Gonzales-Castañeda J, RuizNieto JE, Mireles-Arriaga AI. 2020. Argemone ochroleu$c a$ : (Papaveraceae), alkaloid potential source for agricultural and medicinal uses. Tropical and subtropical agroecosystems 23 (31): In press.

Isman MB, Seffrin R. 2014. Natural insecticides from the Annonaceae: A unique example for developing biopesticides. In: Singh D, editor. Advances in Plant Biopesticides. New Delhi, Springer. P. 21-33.

León-López L, Guzmán-Ortíz DLA, García Berumen JA, Chávez Marmolejo CG, Peña-Cabriales JJ. 2014. Consideraciones para mejorar la competitividad de la región "El Bajío" en la producción nacional de fresa. Revista Mexicana de Ciencias Agrícolas 5(4): 673-686.

López-Medina J, López-Aranda JM, Medina-Míngez JJ, Miranda-Enamorado L, Soria-Navarro C, DomínguezRomero FJ, Vázquez-Ortiz E, Flores-Gil F. 2007. Strawberry production from transplants fumigated with methyl bromide alternatives. Spanish Journal of Agricultural Research (3): 407-416.

Mariscal-Amaro LA, Rivera-Yerena A, Dávalos-González PA, Ávila-Martínez D. 2017. Situación actual de hongos asociados a la secadera de la fresa (Fragaria $\times a n a n a s s a$ duch.) en Guanajuato, México. Agrociencia 51(6): 673-681. 
Martínez AM, Aguado-Pedraza AJ, Viñuela E, RodríguezEnríquez CL, Lobit P, Gómez B, Pineda S. 2017. Effects of ethanolic extracts of Argemone ochroleuca (Papaveraceae) on the food consumption and development of Spodoptera frugiperda (Lepidoptera: Noctuidae). Florida Entomologist 100(2): 339-345. https://doi.org/10.1653/024.100.0232

Mdee LK, Masoko P, Eloff JN. 2009. The activity of extracts of seven common invasive plant species on fungal phytopathogens. South African Journal of Botany 75(2): 375-379. https://doi.org/10.1016/j.sajb.2009.02.003

Nelson PE, Toussoun TA, Marasas WFO. 1983. Fusarium Species: An Illustrated Manual for Identification. Penn State University Press. University Park, USA.

Ochoa YM, Cerna E, Landeros J, Hernández S, Delgado JC. 2012. Evaluación in vitro de la actividad antifúngica de cuatro extractos vegetales metanólicos para el control de tres especies de Fusarium spp. Phyton 81(1): 69-73.

Pedraza RO, Motok J, Salazar SM, Ragout A, Mentel MI, Tortora ML, Guerrero-Molina MF, Wini BB, Díaz-Ricci JC. 2010. Growth-promotion of strawberry plants inoculated with Azospirillum brasilense. World Journal of Microbiology and Biotechnology 26(2): 265-272. https://doi.org/10.1007/s11274-009-0169-1

Rizvi R, Mahmood I, Tiyagi SA, Khan Z. 2012. Effect of some botanicals for the management of plant-parasitic nematodes and soil-inhabiting fungi infesting chickpea. Turkish Journal of Agriculture and Forestry 36(6): 710-719. https://doi.org/10.3906/sag-1208-12

Roberts MF, Wink M. 1998. Alkaloids: Biochemistry, Ecology, and Medicinal Applications. Springer Science Business Media. New York, USA.

Rzedowski C, Rzedowski G. 2001. Flora fanerogámica del Valle de México. Instituto de Ecología/ Comisión Nacional para el Conocimiento y Uso de la Biodiversidad. Michoacán, México.

[SAS] Statistical Analysis Systems. [internet]. 2002. SAS/ STAT Users guide, version 9. SAS Institute Inc. North Carolina, USA.

Sharma A, del Carmen Flores-Vallejo R, Cardoso-Taketa A, Villarreal ML. 2017. Antibacterial activities of medicinal plants used in Mexican traditional medicine. Journal of ethnopharmacology 208: 264-329. https:// doi.org/10.1016/j.jep.2016.04.045

[SIAP] Servicio de Información Agroalimentaria y Pesquera. [internet]. 2018. Resumen nacional por estado. [cited 2019 Nov 01]. Disponible en: http:// infosiap.siap.gob.mx:8080/agricola_siap_gobmx/ ResumenProducto.do.

SiddiquiIA,ShaukatSS, KhanGH,ZakiMJ.2002.Evaluation of Argemone mexicana for control of root-infecting fungi in tomato. Journal of Phytopathology 150(6): 321329. https://doi.org/10.1046/j.1439-0434.2002.00762.x

Singh A, Singh S, Singh S, Singh T, Singh V, Pandey V, Singh U. 2009. Fungal spore germination inhibition by alkaloids dehydrocorydalmine and oxyberberine. Journal of Plant Protection Research 49(3): 287-289. https://doi.org/10.2478/v10045-009-0046-9

Singh S, Singh A, Keshariwala M, Singh TD, Singh VP, Pandey VB, Singh UP. 2010. The mixture of tertiary and quaternary alkaloids isolated from Argemone ochroleu$c a$ inhibits spore germination of some fungi. Archives of Phytopathology and Plant Protection 43(13): 12491253. https://doi.org/10.1080/03235400802404627

Tortora ML, Díaz-Ricci JC, Pedraza RO. 2012. Protection of strawberry plants (Fragaria ananassa Duch.) against anthracnose disease induced by Azospirillum brasilense. Plant and Soil 356(1): 279-290. https://doi.org/10.1007/ s11104-011-0916-6

Villa-Martínez A, Pérez-Leal R, Morales-Morales HA, Basurto-Sotelo M, Soto-Parra JM, Martínez-Escudero E. 2015. Situación actual en el control de Fusarium spp. y evaluación de la actividad antifúngica de extractos vegetales. Acta Agronómica 64(2): 194-205. https://doi. org/10.15446/acag.v64n2.43358

White TJ, Burns T, Lee S, Taylor J. 1990. Amplification and direct sequencing of fungal ribosomal RNA genes for phylogenetics. In: Innis MA, Gelfand DH, Sninsky JJ, White TJ, editors. PCR Protocol: A Guide to Methods and Applications. San Diego, Academic Press. P. 315-322.

Zhang D, Yan D, Fang W, Huang B, Wang X, Wang X, Wang Q. 2019. Chloropicrin alternated with biofumigation increases crop yield and modifies soil bacterial and fungal communities in strawberry production. Science of the Total Environment 675: 615-622. https:// doi.org/10.1016/j.scitotenv.2019.04.222. 